\title{
Effects of thermophoresis on mixed convection flow from a moving vertical plate in a parallel free stream in the presence of thermal radiation
}

\author{
P. M. Patil \\ Head of the Department of Mathematics, JSS's Banashankari Arts, Commerce and S. K. Gubbi Science College, \\ Vidyagiri, Dharwad - 5800 004, India.
}

\begin{abstract}
This article is focused on the numerical investigation of a steady two-dimensional mixed convection boundary layer flow along a moving semi-infinite vertical plate. The plate is assumed to move with a constant velocity in the direction of the flow. The influences of thermophoresis and the thermal radiation are also included in the analysis. The governing boundary layer equations for the flow, thermal and species concentration fields are transformed into a non-dimensional form by a group of non-similar transformations. The resulting system of differential equations is solved by an implicit finite difference scheme in coupled highly nonlinear partial combination with the quasi-linearization technique. The effects of various parameters on the velocity and species concentration profiles and the wall thermophoretic deposition velocity are presented in terms of graphs. The present results are compared with previously published work and are found to be in excellent agreement.
\end{abstract}

Keywords: Thermophoresis; mixed convection; parallel free-stream; thermal radiation; finite difference.

\section{Introduction}

Thermophoresis is a phenomenon in which the motion of suspended particles in a fluid induced by a temperature gradient is of prime practical importance in a variety of industrial and engineering applications including aerosol collection (thermal precipitators), nuclear reactor safety, gas cleaning, corrosion of heat exchangers, and micro-contamination control. Further, this phenomenon has many useful practical applications in removing small particles from gas streams, in determining exhaust gas particle trajectories from combustion devices, and in studying the particulate material deposition on turbine blades. It has also been shown that thermophoresis is the dominant mass transfer mechanism in the modified chemical vapor deposition process used in the fabrication of optical fiber perform and is also important in view of its relevance to postulated accidents by radioactive particle deposition in nuclear reactors. In many industries the composition of processing gases may contain any of an unlimited range of particle, liquid, or gaseous contaminants and may be influenced by uncontrolled factors of temperature and humidity. When such an impure gas is bounded by a solid surface, a boundary layer will develop, and energy and momentum transfer gives rise to velocity and temperature gradients. Mass transfer caused by gravitation and molecular diffusion, eddy diffusion and inertial impact results in deposition of the suspended components onto the surface. In the application of pigments, or chemical coating of metals, or removal of particles from a gas stream by filtration, there can be distinct advantages in exploring deposition mechanisms to improve efficiency.

Goren [1] was the one of the pioneers to study the role of thermophoresis in laminar flow of viscous and incompressible fluid. He used the classical problem of flow over a flat plate to calculate deposition rates and showed that substantial changes in surface depositions can be obtained by increasing the difference between the surface and free stream temperatures. This was later followed by similarity solutions of two dimensional laminar boundary layers and stagnation point flows by Gokoglu and Rosner [2], and Park and Rosner [3]. Similarity solutions were also obtained by Chiou [4] for the problem of a continuously moving surface in a stationary incompressible fluid, including the combined effects of convection, diffusion, wall velocity and thermophoresis. The thermophoretic deposition of small particles in forced convection laminar flow over inclined plates was discussed by Garg and Jayaraj [5]. Alam et al. [6] studied the effects of variable suction and thermophoresis on steady MHD mixed convection flow, heat and mass transfer over a semi-infinite permeable inclined plate in the presence of thermal radiation. Recently, Bakier and Gorla [7] have investigated the effects of thermophoresis and radiation on laminar flow along a semi-infinite vertical plate.

The understanding of the boundary layer behavior over a moving vertical plate in a parallel free stream is very important because it has many important practical applications such as the aerodynamic extrusion of plastic sheets, the cooling of an infinite metallic plate in a cooling bath, the boundary layer along material handling conveyers, the boundary layer along a liquid film in condensation processes, paper production etc. The analysis of boundary layer flow induced by a moving flat surface in an otherwise ambient fluid was first initiated by 
Sakiadis [8] by both analytical and numerical schemes. Remarkable results were found between this analysis and the analysis of the boundary layer on a stationary plate in a moving fluid presented by Blasius [9]. In fact, the skin friction coefficient is approximately $30 \%$ higher for the Sakiadis [8] case compared to the Blasius case [9]. Tsou et al. [10] have showed experimentally that the flow and heat transfer problem from a continuously moving surface is a physically realizable one and performed investigations on its basic characteristics. Abdelhafez [11] examined the boundary layer flow on a moving flat plate in a parallel stream. He showed that Blasius and Sakiadis problems are two special cases of his problem.

Chappidi and Gunnerson [12] have studied the laminar boundary layer in two cases $U_{w}>U_{\infty}$ and $U_{\infty}>U_{w}$ separately and formulated two sets of boundary value problems. Afzal et al. [13] formulated a single set of boundary conditions by employing the composite reference velocity $U=U_{w}+U_{\infty}$, where $U_{w}$ is the moving plate velocity and $U_{\infty}$ is the free stream velocity, instead of considering $U_{w}$ and $U_{\infty}$ separately as done by Abdelhafez [11], irrespective of whether $U_{w}>U_{\infty}$ or $U_{\infty}>U_{w}$. Lin and Haung [14] have analyzed for horizontal isothermal plate moving in parallel or reversibly to a free stream where similarity and nonsimilarity equations are used to obtain the flow and thermal fields. The detailed analysis of the flow field considering the combined motions of a bounding surface and free stream in the same direction have been discussed by Abraham and Sparrow [15], and Sparrow and Abraham [16].

Karwe and Jaluria $[17,18]$ have presented mixed convection flow over a continuous plate moving at a uniform speed in material processes, such as hot rolling, extrusion and drawing etc. Ingham [19] investigated the existence of the singular and non-unique solutions for the free convection boundary layer flow near a continuously moving vertical plate with temperature inversely proportional to the distance along the plate. Ali and Al-Yousef [20] have discussed the problem of laminar mixed convection flow adjacent to a uniformly moving vertical plate with suction or injection. The steady laminar flow and heat transfer characteristics of a continuously moving vertical sheet of extruded material are studied close to and far downstream from the extrusion slot by Al-sanea [21]. Soundalgekar and Murty [22] have discussed the effects of power law surface temperature variation on the heat transfer from a continuous moving surface with constant surface velocity. The effects of variable surface temperature and linear surface stretching were investigated by Grubka and Bobba [23]. Similarity solutions were reported by Ali [24] for the case of power-law surface velocity and three different thermal boundary conditions. Ali [25] has extended his work for the stretching surface subject to suction or injection. Moutsoglou and Chen [26] have considered buoyancy effects on flow and heat transfer from an inclined continuous sheet with either uniform wall temperature or uniform surface heat flux.

More recently, Cortell [27] extended the work of Afzal et al. [13], by taking onto account viscous dissipation effects in the energy balance. The effects of transpiration on the flow and heat transfer over a moving permeable surface in a parallel stream are analyzed by Ishak et al [28]. Ishak et al [29] examined the boundary layer flow over a continuously moving thin needle in a parallel stream. Patil [30] discussed the effects of free convection on the oscillatory flow of a polar fluid through a porous medium in presence of variable wall heat flux. Patil and Hiremath [31] have studied the effects of couple stresses on the flow through a porous medium. The development of the boundary layer on a fixed or moving surface parallel to a uniform free stream in presence of surface heat flux has been investigated by Ishak et al [32]. Effects of radiation on the flow and heat transfer over a moving plate in a parallel stream examined by Anuar Ishak [33]. Very recently, Patil et al. [34] analyzed the role of internal heat generation or absorption on flow and heat transfer over a moving vertical plate in a parallel free stream. The comprehensive analysis on steady mixed convection flow due to a moving semiinfinite vertical plate in a parallel free stream with thermal diffusion is also important to be investigated and such studies are yet to appear in the literature.

Furthermore, steady mixed convection flows do not necessarily posses similarity solutions in many flow patterns. The nonsimilarity in such flows may be due to the free stream velocity or due to the curvature of the body or due to the surface mass transfer or even possibly due to all these effects. A solution is called self-similar if a system of partial differential equations can be reduced to a system of ordinary differential equations. If the similarity transformations are only able to reduce the number of independent variables, then the transformed equations are called as semi-similar and the corresponding solutions are the semi-similar solutions. Therefore, an attempt made towards the development of investigation of mixed convection flows, it is of prime importance as well as useful to study the steady mixed convection boundary layer flow along a semi-infinite vertical moving plate in a parallel free stream to obtain non-similar solutions.

In this paper our main goal is to analyze the combined effects of buoyancy and thermophoresis on a steady mixed convection flow from a semi-infinite vertical plate in presence of thermal radiation. The plate is supposed to move parallel to the free stream velocity. The coupled non-linear partial differential equations governing the flow have been solved numerically using an implicit finite difference scheme in combination with 
the quasi-linearization technique [35-37]. In order to verify the correctness of the present investigation, numerical results are compared with previous results available in the open literature.

\section{Mathematical formulation}

We consider a steady laminar viscous and incompressible mixed convection boundary layer flow along a semi-infinite vertical plate moving with velocity $U_{W}$ in the x-direction. The x-axis is taken along the plate in the vertically upward direction and the $y$ - axis is taken normal to it. It is assumed that the viscous dissipation and heat generation during thermophoresis be neglected. The buoyancy force arises due to the temperature difference in the fluid. The fluid is considered to be gray; absorbing-emitting radiation but non-scattering medium and the Rosseland approximation [38] is used to describe the radiative heat flux in the $\mathrm{x}$ - direction is considered negligible in comparison to the y- direction. All thermo-physical properties of the fluid in the flow model are assumed constant except the density variations causing a body force in the momentum equation. The Boussinesq approximation is invoked for the fluid properties to relate density changes, and to couple in this way the temperature field to the flow field [39]. Under these assumptions, the dimensional equations of conservation of mass, momentum, energy and concentration governing the steady mixed convection boundary layer flow over a moving vertical plate are given by:

$$
\begin{aligned}
& \frac{\partial u}{\partial x}+\frac{\partial v}{\partial y}=0 \\
& u \frac{\partial u}{\partial x}+v \frac{\partial u}{\partial y}=v \frac{\partial^{2} u}{\partial y^{2}}+g\left[\beta\left(T-T_{\infty}\right)+\beta^{*}\left(C-C_{\infty}\right)\right] \\
& u \frac{\partial T}{\partial x}+v \frac{\partial T}{\partial y}=\alpha \frac{\partial^{2} T}{\partial y^{2}}-\left(\frac{1}{\rho C_{p}}\right) \frac{\partial q_{r}}{\partial y}, \\
& u \frac{\partial C}{\partial x}+v \frac{\partial C}{\partial y}=D \frac{\partial^{2} C}{\partial y^{2}}-\frac{\partial}{\partial y}\left(V_{T} C\right),
\end{aligned}
$$

The physical boundary conditions are given by

$$
\begin{array}{ll}
y=0: u=U_{w}, \quad v=0, & T=T_{W}, \quad C=C_{W}, \\
y \rightarrow \infty: u \rightarrow U_{\infty}, & T \rightarrow T_{\infty}, T \rightarrow T_{\infty} .
\end{array}
$$

Where, $u$ and $v$ are the fluid velocity components along the $\mathrm{x}$ - and $\mathrm{y}$ - axes; $\mathrm{g}$ the acceleration due to gravity; $U_{W}$ the moving plate velocity; $U_{\infty}$ the free stream velocity; $T$ the temperature of the fluid in the boundary layer; $T_{W}$ the temperature of the wall; $T_{\infty}$ the ambient temperature; $C$ the species concentration in the boundary layer; $C_{W}$ the species concentration at the wall; $C_{\infty}$ the ambient species concentration; $\rho, v, \alpha, D$ the density; the kinematic viscosity; thermal diffusivity and the Brownian diffusion coefficient, respectively; $\beta$ and $\beta^{*}$ the coefficients of thermal expansions of temperature and concentration, respectively; and $C_{P}$ the specific heat at constant pressure. The effect of thermophoresis is usually prescribed by means of an average velocity which a particle acquire when exposed to temperature gradient. However, in the boundary flow, the temperature gradient in the $y$ - direction is very much larger than in the $\mathrm{x}$ - direction, and therefore only the thermophoretic velocity in $\mathrm{y}$ - direction is considered.

The radiation heat flux $q_{r}$ under Rosseland approximation [38], we take

$q_{r}=-\frac{4 \sigma_{S B}}{3 a_{R}} \frac{\partial T^{4}}{\partial y}$

where $\sigma_{S B}$ is the Stefan-Boltzmann constant and $a_{R}$ is the Rosseland mean spectral absorption coefficient.

We assume that the temperature difference within the flow are sufficiently small such that $T^{4}$ can be expressed as a linear function of temperature. We now expand $T^{4}$ in a Taylor series about $T_{\infty}$ as follows:

$$
T^{4}=T_{\infty}^{4}+4 T_{\infty}^{3}\left(T-T_{\infty}\right)+6 T_{\infty}^{2}\left(T-T_{\infty}\right)^{2}+--------
$$

Neglecting higher order terms in the above equation beyond the first degree in $\left(T-T_{\infty}\right)$, we get 
$T^{4} \cong-3 T_{\infty}^{4}+4 T_{\infty}^{3} T$.

In view of Eqs. (6) and (8), the Eq. (3) becomes

$u \frac{\partial T}{\partial x}+v \frac{\partial T}{\partial y}=\alpha\left(1+\frac{16 \sigma_{S B} T_{\infty}^{3}}{3 k a_{R}}\right) \frac{\partial^{2} T}{\partial y^{2}}$.

As a consequence, the thermophoretic velocity $V_{T}$, which appears in Eq. (4), can be expressed in the following form as:

$V_{T}=-\frac{Q v}{T} \frac{\partial T}{\partial y}$,

where $Q$ is the thermophoretic coefficient.

Eq. (4) becomes in view of Eq. (10),

$u \frac{\partial C}{\partial x}+v \frac{\partial C}{\partial y}=D \frac{\partial^{2} C}{\partial y^{2}}+\frac{Q v}{T}\left\{\frac{\partial C}{\partial y} \frac{\partial T}{\partial y}+C \frac{\partial^{2} T}{\partial y^{2}}-\frac{C}{T}\left(\frac{\partial T}{\partial y}\right)^{2}\right\}$.

Applying the following transformations:

$\xi=\left(\frac{U x}{v}\right)^{1 / 2}, \eta=\left(\frac{U}{v x}\right)^{1 / 2} y, \quad \psi(x, y)=(v U x)^{1 / 2} f(\xi, \eta), u=\frac{\partial \psi}{\partial y}, v=-\frac{\partial \psi}{\partial x}$,

$u=U f_{\eta}=U F, U=U_{\infty}+U_{w}, v=-\frac{(v x U)^{1 / 2}}{2 x}\left\{f(\xi, \eta)+2 \xi f_{\xi}-\eta f_{\eta}\right\}$,

$T-T_{\infty}=\left(T_{W}-T_{\infty}\right) G(\xi, \eta),\left(C-C_{\infty}\right)=\left(C_{W}-C_{\infty}\right) H(\xi, \eta) ;$

to Eqs. (1) - (2), (9) and (11), we find that Eq. (1) is satisfied identically. Then Eqs. (2), (9) and (11) become

$F_{\eta \eta}+\frac{f}{2} F_{\eta}+\lambda(G+N H)=\xi\left\{F F_{\xi}-f_{\xi} F_{\eta}\right\}$,

$G_{\eta \eta}+\left(\frac{\operatorname{Pr}}{1+R}\right) \frac{f}{2} G_{\eta}=\xi\left(\frac{\operatorname{Pr}}{1+R}\right)\left\{F G_{\xi}-f_{\xi} G_{\eta}\right\}$,

$H_{\eta \eta}+\frac{S c f}{2} H_{\eta}+\frac{S c Q}{G+\Delta}\left\{H_{\eta} G_{\eta}+H G_{\eta \eta}-\frac{H G_{\eta}^{2}}{G+\Delta}\right\}=S c \xi\left\{F H_{\xi}-f_{\xi} H_{\eta}\right\}$,

where $f=\int_{0}^{\eta} F d \eta+f_{w} ; f_{w}=0$.

The corresponding boundary conditions are:

$\begin{array}{llll}F=1-\varepsilon, & G=1, & H=1, & \text { at } \eta=0, \\ F \rightarrow \varepsilon, & G \rightarrow 0, & H \rightarrow 0, & \text { as } \eta \rightarrow \infty .\end{array}$

Where, $f$ the dimensionless stream function; $F$ the dimensionless velocity; $G$ the dimensionless temperature; $H$ the dimensionless species concentration; $\operatorname{Pr}=v / \alpha$ is the Prandtl number, $S c=v / D$ is the Schmidt number, $\Delta=T_{\infty} /\left(T_{W}-T_{\infty}\right)$ is the thermophoresis parameter, $\lambda$ is the mixed convection parameter and $N$ is the dimensionless parameter representing the ratio between the thermal and the concentration buoyancy forces, which are defined as

$$
\lambda=\frac{G r_{x}}{\operatorname{Re}_{x}^{2}}, \quad N=\frac{G r_{x}^{*}}{G r_{x}} .
$$

Here the parameter, $G r_{x}=g \beta\left(T_{w}-T_{\infty}\right) x^{3} / v^{2}$, is the local thermal Grashof number, $G r_{x}^{*}=g \beta^{*}\left(C_{w}-C_{\infty}\right) x^{3} / v^{2}$ is the local solutal Grashof number and $\operatorname{Re}_{x}=U x / v$ is the local Reynolds number. It should be mentioned that $\lambda>0\left(T_{w}>T_{\infty}\right)$ corresponds to an assisting flow, while $\lambda<0\left(T_{w}<T_{\infty}\right)$ corresponds to an opposing flow and $\lambda=0\left(T_{w}=T_{\infty}\right)$ corresponds to the forced 
convection flow case. Also $N>0$ for the combined buoyancy forces are driving the flow, $N<0$ for the buoyancy forces opposing each other, $N=0$ for no buoyancy effect due to mass diffusion, $N=1$ for the thermal and mass buoyancy forces of the same strength and $N=\infty$ for no buoyancy effect due to thermal diffusion and $\varepsilon=U_{\infty} /\left(U_{\infty}+U_{W}\right)$ corresponds to the ratio of free stream velocity to the composite reference

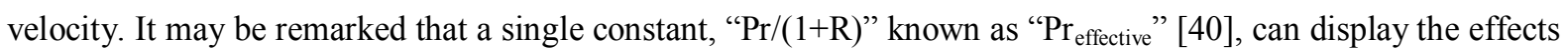
of radiation (R) and Prandtl number $(\operatorname{Pr})$ when the sole interest is to study the effects of radiation (R) and Prandtl number (Pr) for a wide range of parameter values. In such situation a single parameter " $\operatorname{Pr} /(1+\mathrm{R})$ " known as "Preffective" [40] could serve the purpose for the whole range of parameter values. Since the present study concerned about the effect of Newtonian heating only for air $(\mathrm{Pr}=0.7)$ and water $(\mathrm{P} \mathrm{r}=7.0)$, numerical results are presented by indicating known $\mathrm{Pr}$ values of air $(\mathrm{Pr}=0.7)$ and water $(\mathrm{Pr}=7.0)$.

Of interest in this investigation are the non-dimensional velocity $F(\xi, \eta)$ and species concentration $H(\xi, \eta)$ profiles as well as the wall thermophoretic deposition velocity $V_{m}$ which is defined by

$V_{m}=-\frac{Q S c}{1+\Delta} \xi G_{\eta}(\xi, 0)$.

\section{Solution Procedure}

The set of dimensionless non-linear coupled partial differential equations (13) - (15) under the boundary conditions (16) have been solved numerically using an implicit finite-difference scheme in combination with the quasi-linearization technique (Bhattacharyya and Layek [35], Patil [36] and Patil et al. [37]). The quasilinearization technique is viewed as a generalized of the Newton-Raphson technique for the functional equations. The advantage of this technique is its quadratic convergence property. An iterative sequence of linear equations is carefully constructed to approximate the nonlinear equations (13) - (15) under the boundary conditions (16) to achieve quadratic convergence. Applying the quasi-linearization technique, the nonlinear coupled partial differential equations (13) - (15) under the boundary conditions (16) are replaced by the following sequence of linear partial differential equations:

$$
\begin{gathered}
F_{\eta \eta}{ }^{i+1}+X_{1}^{i} F_{\eta}{ }^{i+1}+X_{2}{ }^{i} F^{i+1}+X_{3}^{i+1} F_{\xi}{ }^{i+1}+X_{4}{ }^{i} G^{i+1}+X_{5}{ }^{i} H^{i+1}=X_{6}{ }^{i} \\
G_{\eta \eta}{ }^{i+1}+Y_{1}^{i} G_{\eta}{ }^{i+1}+Y_{2}^{i} G_{\xi}{ }^{i+1}+Y_{3}^{i} F^{i+1}=Y_{4}^{i} \\
H_{\eta \eta}{ }^{i+1}+Z_{1}^{i} H_{\eta}{ }^{i+1}+Z_{2}{ }^{i} H^{i+1}+Z_{3}^{i} H_{\xi}{ }^{i+1} Z_{4}^{i} F^{i+1}+Z_{5}{ }^{i} G_{\eta \eta}{ }^{i+1}+Z_{6}{ }^{i} G_{\eta}{ }^{i+1}+Z_{7}{ }^{i} G^{i+1}=Z_{8}{ }^{i}
\end{gathered}
$$

The coefficient functions with iterative index $i$ are known and the functions with iterative index $(i+1)$ are to be determined. The corresponding boundary conditions are given by

$$
\begin{array}{lll}
F^{i+1}(\xi, 0)=1-\varepsilon & G^{i+1}(\xi, 0)=1, & H^{i+1}(\xi, 0)=1, \\
F^{i+1}(\xi, \eta)=\varepsilon, & G^{i+1}(\xi, \eta)=0, & H^{i+1}(\xi, \eta)=0, \quad \text { at } \quad \eta=\eta_{\infty},
\end{array}
$$

where $\eta_{\infty}$ represents the edge of the boundary layer.

Since the method is presented in detail for partial differential equations in a recent study by Bhattacharyya and Layek [35] and Patil [36] and Patil et al. [37], its detailed description is not provided here. At each iteration step, the sequence of linear partial differential equations (19) - (21) were expressed in finite difference form using central difference scheme in the $\eta$-direction and backward difference scheme in the $\xi$ direction. Thus in each step, the resulting equations were then reduced to a system of linear algebraic equations with a block tri-diagonal matrix, which is solved by Varga's algorithm [41]. To ensure the convergence of the numerical solution to the exact solution, the step sizes, $\Delta \eta$ and $\Delta \xi$ are optimized and taken to be 0.005 and 0.001 , respectively. The presented results are independent of the step sizes up to the fourth significant figure. A convergence criterion based on the relative difference between the current and the previous iteration values is employed. The solution is assumed to have converged and the iteration process terminated once the following convergence criterion is met:

$$
\operatorname{Max}\left\{\left|\left(F_{\eta}\right)_{w}^{k+1}-\left(F_{\eta}\right)_{w}^{k}\right|,\left|\left(G_{\eta}\right)_{w}^{k+1}-\left(G_{\eta}\right)_{w}^{k}\right|,\left|\left(H_{\eta}\right)_{w}^{k+1}-\left(H_{\eta}\right)_{w}^{k}\right|\right\}<10^{-4} .
$$

\section{Results and Discussion}


The computations have been carried out for various values of, $\lambda(-0.18 \leq \lambda \leq 3.0), \varepsilon(0.1 \leq \varepsilon \leq 0.9)$, $N(-0.5 \leq N \leq 1.0), \quad S c(0.22 \leq S c \leq 2.57), \quad \Delta(0 \leq \Delta \leq 10), \quad Q(1 \leq Q \leq 50)$, $R(0 \leq R \leq 1)$ and $\xi(0 \leq \xi \leq 2)$. The edge of the boundary layer $\eta_{\infty}$ has been taken between 5.0 and 12.0 depending on the values of the parameters. Afzal et al. [13] have discussed only self-similar solutions where all solutions along streamwise direction were made congruent using similarity transformations. Governing equations were finally reduced to a set of ordinary differential equations. In such cases, single asymptotic solution will not be able to represent all physical solutions. Further, it is reported by many other investigators also that there are dual solutions with different asymptotic nature. In contrast, authors have captured non-similar solutions at each streamwise location in different time-step by solving coupled set of partial differential equations. In this investigation, authors have observed a single non-similar solution at each streamwise location. Accuracy of the presented approach is verified by direct comparison with the results previously reported by Tsou et al. [10], Soundalgekar and Murty [22], Ali [24], Moutsoglou and Chen [26] and Patil et al. [32]. The results of this comparison are presented in Table 1 and are found to be in excellent agreement.

Figures 1 and 2 display the effects of varying the mixed convection parameter $\lambda$ and the Schmidt number $S c$ on the velocity $F(\xi, \eta)$ and species concentration $H(\xi, \eta)$ profiles with $\operatorname{Pr}=0.7, \Delta=10, \mathrm{~N}=$ $1, \varepsilon=0.5, \xi=0.5, Q=1$ and $R=1$. In the case of buoyancy-aiding flow $(\lambda>0)$, the buoyancy force shows the overshoot in the velocity profiles near the surface for both fluids of smaller $(S c=0.66)$ and higher
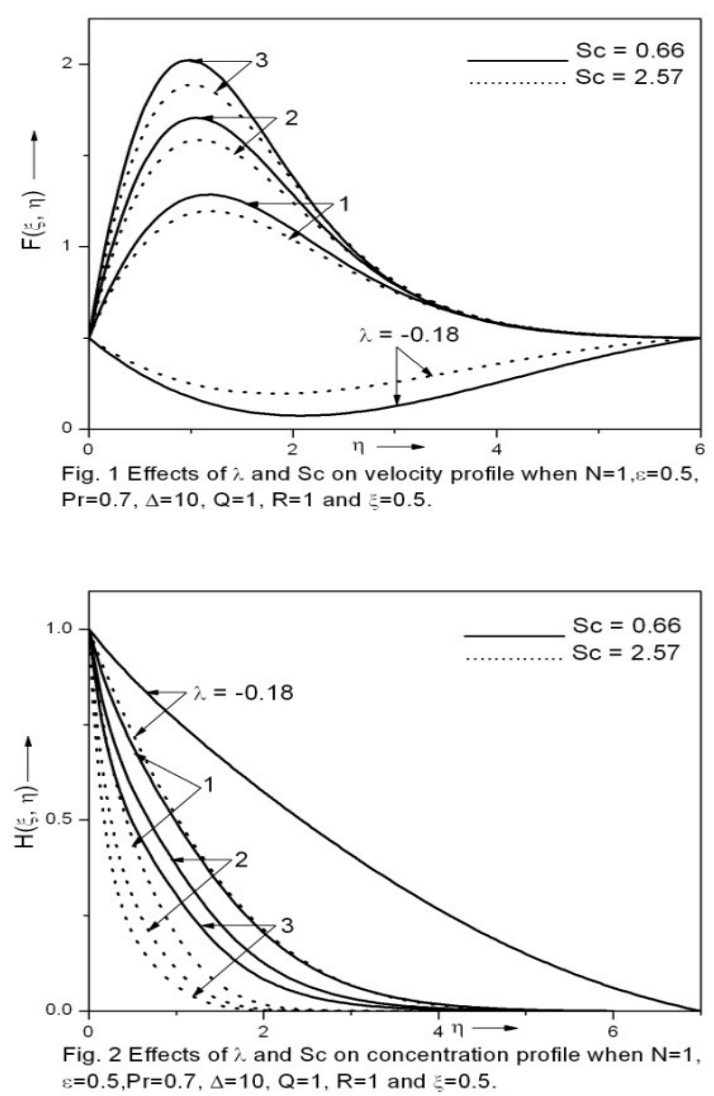

$(S c=2.57)$ values of $S c$, but for higher values $(S c=2.57)$, the overshoot of the velocity profiles is reduced, compared to smaller $(S c=0.66)$ values of $S c$ as shown in Fig. 1. The physical reason is that the lower $S c$ values may mean the lower viscosity of the fluid which increases the local velocity everywhere within the moving boundary layer. Thus, the velocity overshoot occurs. For the higher values of $S c$ the overshoot is reduced because fluids with higher values of the $S c$ imply more viscous fluids, which has less impact on the buoyancy parameter $\lambda$. However, the influence of the parameter $\lambda$ is more prominent for the lower value of $S c(=0.66)$. Fig. 2 also indicates that the magnitude of the species concentration decreases remarkably for 
higher values of the $S c(=2.57)$ as compared with the lower value of $S c(=0.66)$. Further, it is very interesting to note from Figs. 1 and 2 that, the buoyancy opposing force $(\lambda<0)$ reduces the magnitude of the velocity profile considerably within the moving boundary layer while the opposite behavior is found in the case of species concentration profile.

The effects of the ratio of buoyancy forces parameter $N$ and the Schmidt number $S c$ on velocity $F(\xi, \eta)$ and species concentration $H(\xi, \eta)$ profiles are displayed in Figs. 3 and 4 when $\operatorname{Pr}=0.7, \Delta=10$, $\lambda=1, \varepsilon=0.5, \xi=0.5, \mathrm{Q}=1$ and $\mathrm{R}=1$. It is observed that the magnitude of velocity profile increases with the increase of the ratio of buoyancy forces parameter $N$ in the both cases for $S c(=0.66)$ and $S c(=2.57)$ (See Fig. 3). The physical reason is that the assisting ratio of buoyancy forces $(N>0)$ implies a favorable pressure gradient, for which the fluid gets accelerated, which results in thicker momentum boundary layer while the opposing buoyancy forces $(N<0)$ imply that the fluid gets decelerated. However, the magnitude of species concentration profile decreases with the increase of the ratio of buoyancy forces parameter $N$ in the both

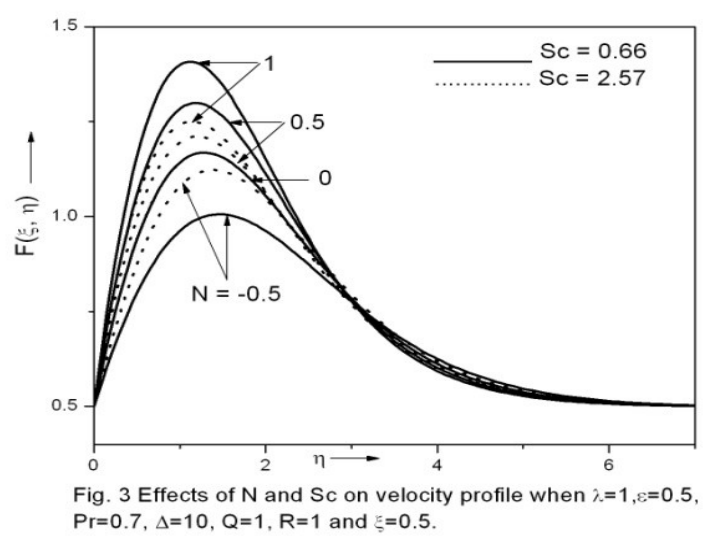

cases for $S c(=0.66)$ and $S c(=2.57)$ (see Fig. 4). The physical reason is that the assisting ratio of buoyancy forces $(N>0)$ acts as a non-favorable pressure gradient due to thermophoresis effect, for which the fluid gets decelerated, which results in the thinner species concentration boundary layer while the opposing ratio of buoyancy forces $(N<0)$ acts as a favorable pressure gradient and for which the fluid gets accelerated. Thus, there is an increase in the concentration boundary layer.

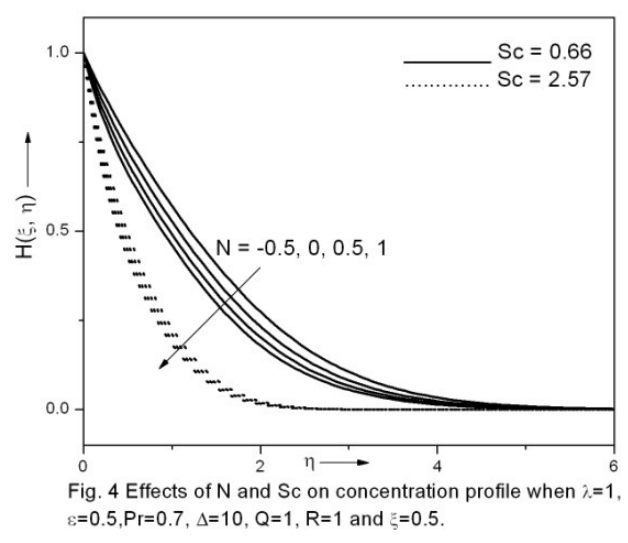

Figure 5 displays the effects of $\varepsilon$ (the ratio of free stream velocity to the composite reference velocity) and the thermophoretic parameter $\Delta$ on the velocity profiles $F(\xi, \eta)$ when $\lambda=1.0, \mathrm{R}=1, \mathrm{~N}=1 \xi=0.5, Q=$ $1, \mathrm{Sc}=2.57$ and $\operatorname{Pr}=0.7$. The velocity profile is strongly depending on $\varepsilon$ because it occurs in the momentum 
equation as well as in the boundary condition for $F(\xi, \eta)$. It has been observed that the magnitude of the velocity profile within the moving boundary layer increases with the increase of $\varepsilon$. The physical reason is that the assisting buoyancy force due to thermal and solutal gradients as well as increase in $\varepsilon$ acts like a favorable pressure gradient which accelerates the fluid for uniform motion causing the velocity overshoots near the surface within the moving boundary layer. However, the magnitude of the velocity profile increases with increasing thermophoretic parameter $\Delta$ when $\varepsilon<0.5$ while it decreases when $\varepsilon>0.5$. It reveals that the thermophoretic effects are crucial for the velocity profile. However, it clearly indicates that the effect of thermophoresis on moving boundary with free stream affects the velocity profile.

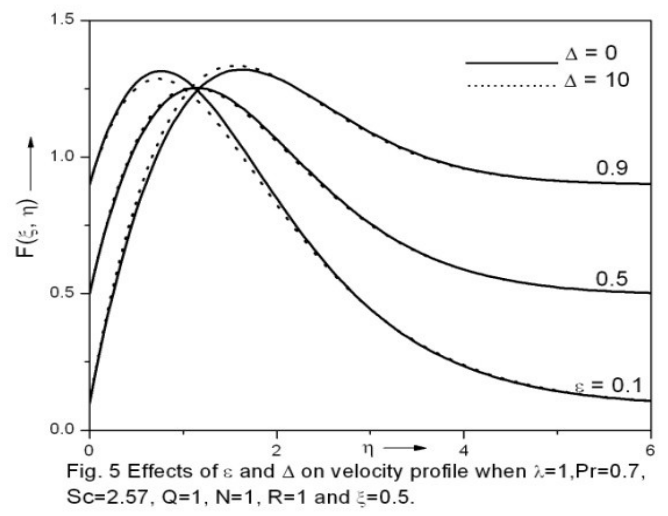

Figure 6 illustrate the role of thermal radiation parameter $R$ and Schmidt number $S c$ on the species concentration profile $H(\xi, \eta)$ with $\operatorname{Pr}=0.7, \Delta=10, \mathrm{~N}=1, \varepsilon=0.5, \xi=0.5, \mathrm{Q}=0.5$ and $\lambda=1$. It is seen that the species concentration profile is substantially influenced by the parameters $R$ and $S c$. The values of the Schmidt number $(S c)$ are chosen to be more realistic, $0.22,0.660 .94,2.57$, representing diffusing chemical species of most common interest like water, Ammonia, Propyl Benzene hydrogen, water vapor and Propyl Benzene, etc., at 25 degree Celsius at one atmospheric pressure. It is observed that the concentration and velocity boundary layers are to decrease as the Schmidt number $S c$ is increased. The physical reason is that the Schmidt number $S c$ leads to a thinning of the concentration boundary layer. As a result the concentration of the

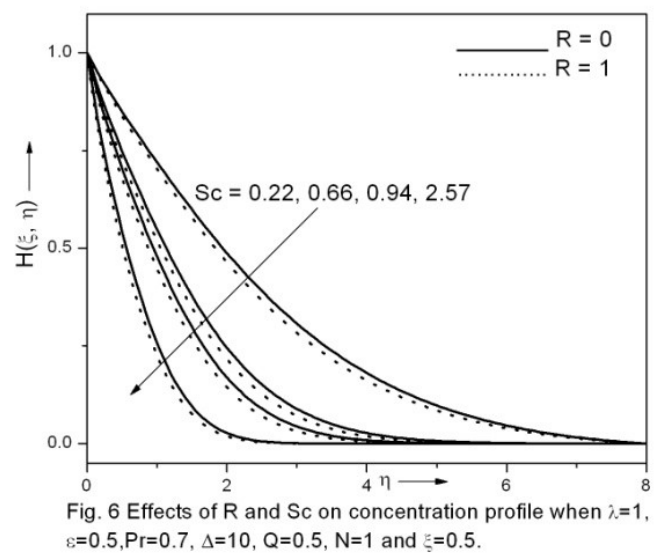

fluid decreases and thus leads to a decrease in the fluid velocity. This is similar to the effect of increasing the Prandtl number $P r$ on the thickness of a thermal boundary layer. Furthermore, species concentration profile $H(\xi, \eta)$ leads to a fall in the magnitude due to the effect of thermal radiation applied at the wall which works as heat source and thus species concentration boundary layer decreases. 


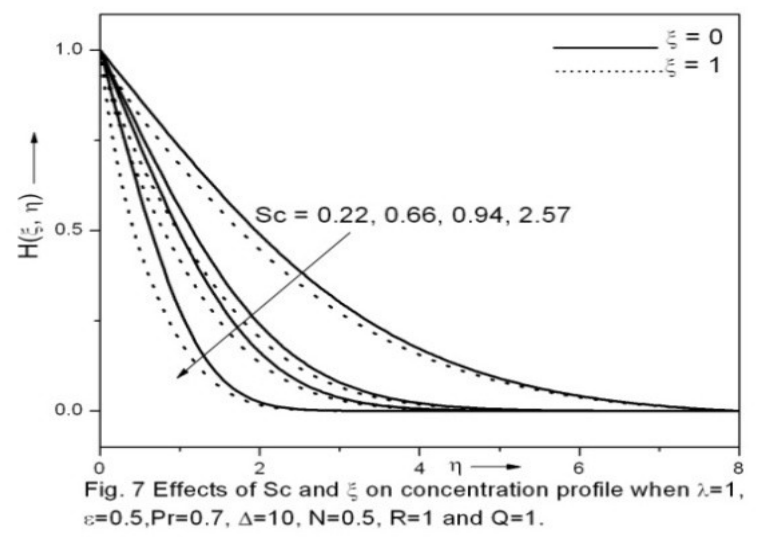

Figure 7 presents the variations of the species concentration profiles $H(\xi, \eta)$ with the non-similar variable $\xi$ and Schmidt number $S c$ for with $\operatorname{Pr}=0.7, \Delta=10, \mathrm{~N}=0.5, R=1 \varepsilon=0.5, \mathrm{Q}=1$ and $\lambda=1$. The profiles drawn at $\xi=0$ represent the similarity solutions when all solutions along the $x$-direction are made congruent using similarity transformations. This figure shows that the concentration profile $H(\xi, \eta)$ decreases with the increase of $\xi$ from 0 to 1 . This clearly indicates that in the thermophoretic flow, an increase in $\xi$ acts as a decelerating force and hence fluid flow gets slower. Thus there is a fall in the magnitude of the species concentration profiles $H(\xi, \eta)$. Also, an increase in $S c$ decreases the mass diffusion and thus, in turn, the magnitude of the species concentration profile $H(\xi, \eta)$ decreases.

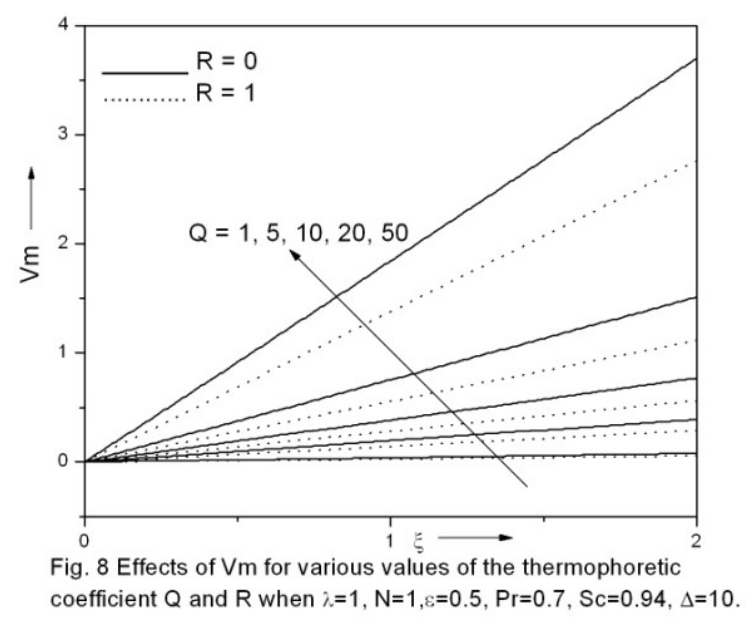

The effects of thermal radiation parameter $R$ on variations of the wall thermophoretic deposition velocity $V_{m}$ against non-similar variable $\xi$ are depicted for various values of thermophoretic coefficient $Q$ in Fig. 8 with $\operatorname{Pr}=0.7, \Delta=10, \mathrm{Sc}=0.94, \mathrm{~N}=1, \varepsilon=0.5$, and $\lambda=1$. It is observed that the values of $V_{m}$ increase with the thermophoretic coefficient $Q$ and non-similar variable $\xi$ steadily. Further, the values of $V_{m}$ is to decrease with the thermal radiation parameter when it increases from 0 to 1 . This indicates that thermal radiation has a pronounced effect on thermophoresis. In particular, for example, the percentage of increase of wall thermophoretic deposition velocity $V_{m}$ by about $97 \%$ and $100 \%$ as thermophoretic coefficient increases from $Q=10$ to $Q=20$ when $R=0$ and $R=1$, respectively, at $\xi=1.0$.

\section{Conclusions}

A numerical investigation has been performed to study the mixed convection boundary layer flow from a moving vertical plate in a parallel free stream in the presence of thermal radiation and thermophoresis. The governing boundary layer equations are transformed into a non-dimensional form by a group of non-similar transformations. The resulting system of coupled non-linear partial differential equations is solved by an implicit 
finite difference scheme in combination with the quasi-linearization technique. The following conclusions have been drawn in the present numerical investigation:

- The velocity profile exhibits significant enhancement for low Schmidt number fluids as compared to the magnitude of the velocity for higher Schmidt number.

- Results reveal that velocity and species concentration boundary layers are to increase with the increase of mixed convection parameter $\lambda$ and the ratio of buoyancy forces $\mathrm{N}$.

- An increase in the stream wise co ordinate $\xi$ acts as a nonfavorable pressure gradient and hence fluid flow gets slower.

- The magnitude of the velocity within the moving boundary layer increases with the increase of $\varepsilon$ (the ratio of free stream velocity to the composite reference velocity).

- The influence of thermal radiation parameter $R$ on the velocity and species concentration profiles is significant and which leads to reduction in the profiles.

- The effects of thermophoretic coefficient leads to an increase in the value of wall thermophoretic deposition velocity $V_{m}$.

\section{Acknowledgement:}

Author acknowledge with thanks to the University Grants Commission, South Western Regional Office Bangalore for financial assistance with grant No. MRP(S)-77/12-13/KAKA060/UGC-SWRO Dated $29^{\text {th }}$ March, 2013. Author also expresses sincere thanks to Dr. Ajith Prasad, Finance Officer, Janata Shikshana Samiti, Dharwad - 580 004, for encouraging the research activities.

Table 1. Comparison of - $G_{\eta}(0)$ for $\lambda=0, \xi=0, \mathrm{R}=0, \varepsilon=0$ and selected values of $\operatorname{Pr}$, the plate being isothermal ( $T_{W}=$ constant $)$ to previously published work.

\begin{tabular}{|c|c|c|c|c|c|c|}
\hline $\operatorname{Pr}$ & 0.7 & 1.0 & 2.0 & 7.0 & 10.0 & 100.0 \\
\hline Tsou et al. [10] & 0.3492 & 0.4438 & -- & -- & 1.6804 & 5.545 \\
\hline $\begin{array}{c}\text { Soundalgekar and } \\
\text { Murty [22] }\end{array}$ & 0.3508 & -- & 0.6831 & -- & 1.6808 & -- \\
\hline Ali [24] & 0.3476 & 0.4416 & -- & -- & 1.6713 & -- \\
\hline $\begin{array}{c}\text { Moutsoglou and } \\
\text { Chen [26] }\end{array}$ & 0.34924 & -- & -- & 1.38703 & -- & -- \\
\hline Patil [34] & 0.35004 & 0.44401 & 0.68314 & 1.38625 & 1.68011 & 5.54610 \\
\hline Present work & 0.35005 & 0.44403 & 0.68316 & 1.38627 & 1.68013 & 5.54612 \\
\hline
\end{tabular}

\section{References:}

[1] S.L. Goren, Thermophoresis of aerosol particles in the laminar boundary layer on a flat surface, Journal of Colloid and Interface Science 61 (1977) 77-85.

[2] S.A. Gokoglu, D.E. Rosner, Thermophoretically augmented mass transfer rate to solid walls across laminar boundary layers, AIA A Journal 24 (1986) 172.

[3] H.M. Park, D.E. Rosner, Combined inertial and thermophoretic effects on particle deposition rates in highly loaded systems, Chemical Engineering Science 44 (1989) 2233-2244.

[4] M.C. Chiou, Effect of thermophoresis on submicron particle deposition from a forced laminar boundary layer flow onto an isothermal moving plate, Acta Mechanica 129 (1998) 219-229.

[5] V. K. Garg, S. Jayaraj, Thermophoresis of aerosol particles in laminar flow over inclined plates, International Journal of Heat and Mass Transfer 31 (1988) 875-890.

[6] M. S. Alam, M. M. Rahman, M. A. Sattar, Effects of variable suction and thermophoresis on steady MHD combined free-forced convective heat and mass transfer flow over a semi-infinite permeable inclined plate in the presence of thermal radiation, International Journal Thermal Sciences 47 (2008) 758-765.

[7] A. Y. Bakier, R. S. R. Gorla, Effects of thermophoresis and radiation on laminar flow along a semi-infinite vertical plate, 47 (2011) 419-765.

[8] H. Blasius, Grenzchiechten in flussigkeiten rnit kleiner reibung, Z. Math. Phys. 56 (1908) 1- 37.

[9] B. C. Sakiadis, Boundary-layer behavior on continuous solid surfaces, II. The boundary layer behavior on continuous flat surface, AIChE J 7 (1967) $221-225$.

[10] F.K. Tsou, E.M. Sparrow, R.J. Goldstein, (1967) Flow and heat transfer in the boundary layer on a continuous moving surface, Int. J. Heat Mass Transfer 10 (1967) 219-235.

[11] T. A. Abdelhafez, Skin friction and heat transfer on a continuous flat surface moving in a parallel free stream, Int. J. Heat Mass Transfer 28 (1985) 1234- 1237. 
[12] P. R. Chappidi, F. S. Gunnerson, Analysis of heat and momentum transport along a moving surface, Int. J. Heat Mass Transfer 32 (1989) 1383- 1386.

[13]. N. Afzal, A. Baderuddin, A. A. Elgarvi, Momentum and heat transport on a continuous flat surface moving in a parallel stream, Int. J. Heat Mass Transfer 36 (1993) 3399-3403.

[14]. H. T. Lin, S. F. Haung, Flow and heat transfer of plane surface moving in parallel and reversely to the free stream, Int. J. Heat Mass Transfer 37 (1994) 333- 336.

[15] J. P. Abraham, E. M. Sparrow, Friction drag resulting from simultaneous imposed motions of a free stream and its bounding surface, Int. J. Heat and Fluid Flow, 26 (2005) 289 - 295.

[16] E. M. Sparrow, J. P. Abraham, Universal solutions for the stream wise variation of the temperature of a moving sheet in the presence of a moving fluid, Int. J. Heat and Mass Transfer, 48 (2005) 3047 - 3056.

[17] M. V. Karwe, Y. Jaluria, Fluid flow and mixed convection transport from a moving plate in rolling and extrusion processes, ASME J. Heat Transfer 110 (1988) 655-661.

[18] M. V. Karwe, Y. Jaluria, Numerical simulation of thermal transport associated with a continuously moving flat sheet in material processing, ASME J. Heat Transfer 113 (1991) 612- 619.

[19] D. B. Ingham, Singular and non-unique solutions of the boundary layer equations for the flow due to free convection near a continuously moving plate, J Applied Math and Phys (ZAMP), 37 (1986) 559- 572.

[20] M. Ali, F. Al-Yousef, Laminar mixed convection from a moving vertical surface with suction or injection, Heat and Mass Transfer 33 (1998) 301- 306.

[21] Sami A. Al- Sanea, Mixed convection heat transfer along a continuously moving heated vertical plate with suction or injection, Int. J. Heat Mass Transfer 47 (2004) 1445-1465.

[22] V.M. Soundalgekar, T.V.R. Murty, Heat transfer in flow past a continuous moving plate with variable temperature, Warme-und Stoffubertragung 14 (1980) 91-93.

[23] L.J. Grubka, K.M. Bobba, Heat transfer characteristics of a continuous, stretching surface with variable temperature, ASME J. Heat Transfer 107 (1985) 248-250.

[24] M.E. Ali, Heat transfer characteristics of a continuous stretching surface, Warme-und Stoffubertragung 29 (1994) $227-234$.

[25] M.E. Ali, On thermal boundary layer on a power-law stretched surface with suction or injection, Int. J. Heat and Fluid Flow 16 (1995) 280-290.

[26] A. Moutsoglou, T.S. Chen, Buoyancy effects in boundary layers on inclined, continuous, moving sheets, ASME J. Heat Transfer 102 (1980) 371-373.

[27] R. Cortell, Flow and heat transfer in moving fluid over a moving flat surface, Theor. Comput. Fluid Dyn. 21 (2007) $435-446$.

[28] A. Ishak, R. Nazar, I. Pop, The effects of transpiration on the flow and heat transfer over a moving permeable surface in a parallel stream, Chemical Engineering Journal, 148 (2008) 63 - 67.

[29] A. Ishak, R. Nazar, I. Pop, Boundary layer flow over a continuously moving thin needle in a parallel stream, Chinese Physics Letters, 24 (2007) 2895 - 2897.

[30] P. M. Patil, Effects of free convection on the oscillatory flow of a polar fluid through a porous medium in presence of variable wall heat flux, Journal of Engineering Physics and Thermophysics 81(5) (2008) 905 - 922.

[31] P. M. Patil and P. S. Hiremath, A note on effects of couple stresses on the flow through a porous medium, Rheologica Acta 31 (1992) $206-207$.

[32] A. Ishak, R. Nazar, I. Pop, Flow and heat transfer characteristics in a moving flat plate in a parallel stream with constant surface heat flux, Heat Mass Transfer, 45 (2009) 563 - 567.

[33] Anuar Ishak, Radiation effects on the flow and heat transfer over a moving plate in a parallel stream, Chinese Physics Letters, 26 (2009) 034701.

[34] P. M. Patil, S. Roy, I. Pop, Flow and heat transfer over a moving vertical plate in a parallel free stream: Role of internal heat generation or absorption, Chem. Eng. Comm., 199 (2012) 658-672.

[35] K. Bhattacharyya, G. C. Layek, Chemically reactive solute distribution in MHD boundary layer flow over a permeable stretching sheet with suction or blowing, Chemical Engineering Communications 197 (12) (2010) 1527- 1540.

[36] P. M. Patil, Effects of surface mass transfer on a steady mixed convection flow from a vertical stretching sheet with variable wall temperature and concentration, Int. J Num. Methods Heat Fluid Flow 22 (2012)287-305.

[37] P. M. Patil, S. Roy, A. J. Chamkha, Mixed convection flow over a vertical power law stretching sheet, Int. J Num. Methods Heat Fluid Flow 20 (2010) 445-458.

[38] Brewster, M. Q., Thermal radiative transfer properties, Wiley, Canada (1992).

[39] Schlichting, H., Boundary Layer Theory, Springer, New York (2000).

[40] E. Magyari, A. Pantokratoras, Note on the effect of thermal radiation in the linearised Rosseland approximation on the heat transfer characteristics of various boundary layer flows, International Communications in Heat and Mass Transfer 38 (2011) $554-556$.

[41] Varga, R.S., Matrix Iterative Analysis, Prentice Hall (2000). 